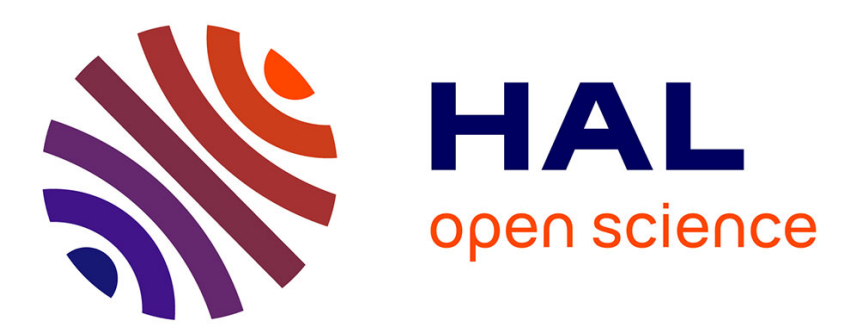

\title{
Impact of inner-core size on the dipole field behaviour of numerical dynamo simulations
}

Florian Lhuillier, Gauthier Hulot, Yves Gallet, Tobias Schwaiger

\section{To cite this version:}

Florian Lhuillier, Gauthier Hulot, Yves Gallet, Tobias Schwaiger. Impact of inner-core size on the dipole field behaviour of numerical dynamo simulations. Geophysical Journal International, 2019, 218 (1), pp.179-189. 10.1093/gji/ggz146 . insu-02376555

\section{HAL Id: insu-02376555 \\ https://hal-insu.archives-ouvertes.fr/insu-02376555}

Submitted on 22 Nov 2019

HAL is a multi-disciplinary open access archive for the deposit and dissemination of scientific research documents, whether they are published or not. The documents may come from teaching and research institutions in France or abroad, or from public or private research centers.
L'archive ouverte pluridisciplinaire HAL, est destinée au dépôt et à la diffusion de documents scientifiques de niveau recherche, publiés ou non, émanant des établissements d'enseignement et de recherche français ou étrangers, des laboratoires publics ou privés. 


\section{INTRODUCTION}

The Earth's solid inner core is thought to have been created by the crystallisation of the fluid outer core as a consequence of secular cooling (Jacobs 1953). This crystallisation is responsible for the production of thermal and compositional buoyancy at the innercore boundary (ICB) because of the joint release of latent heat and light elements, thermal buoyancy being also produced by secular cooling or internal heating. The relative size of the growing inner core can be quantified by its aspect ratio $\chi=r_{i} / r_{0}$, where $r_{i}$ and $r_{0}$ are the inner and outer core radii respectively. For the presentday geodynamo $(\chi=0.35)$, it is thought that the principal driving mechanism of the convection is the release of light elements at the ICB, whereas secular cooling and internal heating acted prior to inner-core nucleation (e.g., Nimmo 2015). The geodynamo evolution is thus largely conditioned by the inner-core history. Due to large uncertainties in the heat flow at the CMB (from 5 to $15 \mathrm{TW}$ approximately) and in the thermal conductivity of the outer core (from 30 to $100 \mathrm{~W} /(\mathrm{Km})$ approximately), the inner core may have nucleated from 2 Gyr ago (e.g., Labrosse et al. 2001) to less than 1 Gyr ago (e.g., Labrosse 2015; Davies 2015; Olson et al. 2015).

One of the most striking features of the Earth's magnetic field is its ability to produce polarity reversals (e.g., Valet \& Fournier 2016). Even though the polarity reversal history is rather well doc- umented during the Phanærozoic (e.g., Biggin et al. 2012), its interpretation is still a matter of debate. One of the biggest mysteries is the existence of prolonged periods ( $>20 \mathrm{Myr}$ ) with stable polarity termed superchrons: three such events are documented during the Phanærozoic (e.g., Pavlov \& Gallet 2005) and several were proposed during the Proterozoic (e.g., Pavlov \& Gallet 2010; Gallet et al. 2012; Driscoll \& Evans 2016). Why reversal frequency varies and why superchrons exist are highly debated questions. On the one hand, the evolution in reversal frequency has been traditionally ascribed to a gradual change in the conditions imposed by the mantle on the core (e.g., McFadden \& Merrill 1984, 2000; Glatzmaier et al. 1999). On the other hand, it was suggested that the occurrence of superchrons could result from sudden transitions ascribable either to rapid changes of boundary conditions at the core-mantle boundary (e.g., Gallet \& Hulot 1997; Courtillot \& Olson 2007) or to a spontaneous non-linear transition of the geodynamo between reversing and stable states (e.g., Hulot \& Gallet 2003). It is also an open question whether the frequency of superchrons evolved over the Earth's history. Coe \& Glatzmaier (2006) proposed that superchrons are more abundant during the Proterozoic than during the Phanærozoic, whereas Driscoll \& Evans (2016) suggested a quasistationary rate of superchrons over the past 2 Gyr. At the opposite extreme, another mystery is the episodes of hyperactivity with a reversal frequency greater than 10 per Myr (e.g., Gallet \& Pavlov 
2016). Two such events may have occurred in the past $600 \mathrm{Myr}$ : one during the Middle Jurassic (e.g., Tivey et al. 2006), one during the Late Ediacaran (e.g., Shatsillo et al. 2015; Bazhenov et al. 2016).

In spite of limited computing capacities that require an excessive viscosity by several orders of magnitude, numerical dynamo simulations in spherical geometry (e.g., Glatzmaier \& Roberts $1995 \mathrm{a}, \mathrm{b})$ have been able to produce "Earth-like" magnetic fields akin to the present-day field (e.g., Glatzmaier 2002; Christensen et al. 2010; Aubert et al. 2017). Some of them have been integrated over the equivalent of tens of Myr to investigate the reversing behaviour of the geodynamo with homogeneous boundary conditions (e.g., Wicht 2005; Olson 2007; Lhuillier et al. 2013; Wicht \& Meduri 2016) or factors possibly controlling the reversal frequency, such as a time-varying CMB heat flow (e.g., Driscoll \& Olson 2011) or time-dependent CMB heat flow heterogeneities (e.g., Olson et al. 2013). The influence of the electrical conductivity of the inner core on the reversal frequency is an unanswered question. Theoretical studies (e.g., Hollerbach \& Jones 1993) suggest that the finite conductivity of the inner core should stabilise the geodynamo and thus reduce reversal frequency. Numerical simulations, however, provide contradictory results, a conducting inner core leading to a reduction in reversal frequency in some cases (e.g., Dharmaraj \& Stanley 2012), an increase in other cases (e.g., Lhuillier et al. 2013) or to no influence at all (e.g., Wicht 2002). The influence of inner-core size has also been explored in numerical dynamo simulations. In a systematic study of a suite of thermally-driven dynamos with $0.25 \leq \chi \leq 0.65$, Heimpel et al. (2005) reported an increase in the number of columnar vortices with $\chi$, leading to an increase in the field strength with $\chi$. To better account for the Earth's history, the evolution of the aspect ratio can also be combined with a thermal evolution model of the Earth (e.g., Roberts \& Glatzmaier 2001; Aubert et al. 2009; Olson et al. 2013; Heimpel \& Evans 2013; Driscoll 2016; Landeau et al. 2017), with the caveat that the thermodynamical parameters suffer from large uncertainties. These models underline in particular the impact of the inner-core nucleation on the evolution of the field intensity. Aubert et al. (2009) predict a strong dipolar field over the whole geodynamo history with some temporal variations and a signature related to inner-core nucleation, which however depend on the exact assumptions used. Such evolutions are not unequivocally observed in the available palaeointensity data spanning the past three billion years (e.g., Biggin et al. 2015; Smirnov et al. 2016). The absence of a clear long-term trend in palaeointensity over this period appears to be in better agreement with the dynamo simulations recently performed by Landeau et al. (2017). These authors further suggest the possibility, prior to inner-core nucleation, of a bistability between a likeliest strong-field dipolar state and a more marginal low-field multipolar state with frequent reversals.

In this study, we do not aim to model the geodynamo's history in a comprehensive way, but mainly focus on a closely related aspect, namely the impact of inner-core size on the dipole field behaviour of numerical dynamo simulations. We base our investigation on a set of 56 chemically-driven dynamos with various aspect ratios $(0.10 \leq \chi \leq 0.44)$, integrated over a sufficiently long time to allow the analysis of the statistical properties of reversals. We present in Section 2 the models and quantities employed. We then develop in Section 3 the key results in terms of field strength, dipolarity, secular variation and reversal frequency. We finally elaborate in Section 4 on a possible application of these results to the Earth's magnetic field.

\section{MODELS AND TOOLS}

\subsection{Dimensionless parameters}

We model the Earth's fluid outer core by a spherical shell of aspect ratio $\chi=r_{i} / r_{0}$, rotating about the $z$-axis with a constant angular velocity $\Omega$, and within which an electrically conducting fluid is chemically convecting. We solve the magnetic induction equation for the magnetic field $\boldsymbol{B}$ within the MHD approximation, the Navier-Stokes and transport equation for the velocity field $\boldsymbol{u}$ and the codensity $C$ within the Oberbeck-Boussinesq approximation (Braginsky \& Roberts 1995). In this study, the codensity $C$ is proportional to the excess concentration in light elements from a well-mixed basic state. We apply no-slip boundary conditions and consider an electrically conducting inner core that rotates subject to viscous and Lorentz torques (Wicht 2002). We finally impose a fixed anomaly of light elements at the inner boundary and a zero flux at the outer boundary.

Following Aubert et al. (2008), length is scaled with the shell depth $D=r_{0}-r_{i}$. Time is scaled with the inverse of the rotation rate $\Omega$. Magnetic field is scaled with $(\rho \mu)^{1 / 2} \Omega D$, where $\rho$ is the fluid density and $\mu$ the magnetic permeability. The codensity is scaled with $|S| / \Omega$, where $S$ is a volumetric sink. In addition to the aspect ratio $\chi$, the four control parameters are the Ekman number $E=\nu /\left(\Omega D^{2}\right)$ that measures the relative importance of viscous to Coriolis forces, the hydrodynamic Prandtl number $\operatorname{Pr}=\nu / \kappa$ that quantifies the ratio between kinematic and chemical diffusivities, the magnetic Prandtl number $P m=\nu / \eta$ that quantifies the ratio between kinematic and magnetic diffusivities, and the modified Rayleigh number $R a=g_{0}|S| /\left(\Omega^{3} D\right)$ that quantifies the vigour of convection. The quantities $\nu, \kappa$ and $\eta$ are respectively the kinematic, chemical and magnetic diffusivities, whereas $g_{0}$ is the gravity at radius $r=r_{0}$. For an Earth with outer core radius $r_{0}$ and a growing inner core, the outer-core thickness varies as $D=r_{0}(1-\chi)$. It is thus of interest to define a modified Ekman number $E^{\star}=\nu /\left(\Omega r_{0}^{2}\right)=(1-\chi)^{2} E$ that is independent of the aspect ratio $\chi$. By analogy, we define a modified Rayleigh number $R a^{\star}=g_{0}|S| /\left(\Omega^{3} r_{0}\right)=(1-\chi) R a$ that is also independent of $\chi$.

The relevant output parameters are: the convective power density $p=\phi /\left(\rho \Omega^{3} D^{2}\right)$ where $\phi$ is the volumetric power density generated by buoyancy forces (e.g., Landeau et al. 2017); the magnetic Reynolds number $R m=U D / \eta$ where $U$ is the time-averaged rms velocity over the outer-core shell; or its variant $R m^{\star}=U r_{0} / \eta=R m /(1-\chi)$ that is independent of the aspect ratio; the Rossby number $R o=U /(\Omega D)$; or its variant $R o^{\star}=U /\left(\Omega r_{0}\right)=R o(1-\chi)$ that is independent of the aspect ratio; the local Rossby number $R o_{\ell}=\ell U /(\pi \Omega D)$ where $\pi / \ell$ is the characteristic half-wavelength of the flow (e.g., Christensen $\&$ Aubert 2006); the dipolarity $f_{\text {dip }}$ defined as the time-averaged rms amplitude of the dipole relative to the field up to degree 12 at the core-mantle boundary; the dynamo efficiency $f_{\text {ohm }}$ defined as the time-averaged fraction of Ohmic dissipation with respect to the convective power (e.g., Christensen \& Aubert 2006). Within the chosen non-dimensionalisation, the dimensionless magnetic field is expressed by the Lehnert number $L e=B /(\Omega D \sqrt{\rho \mu})$, where $B$ is the time-averaged rms magnetic field over the outer-core shell. In order to remain independent of the aspect ratio, the temporal quantities depending on diffusion are expressed in units of the dipole decay time $\tau_{\text {dip }}=r_{0}^{2} /\left(\pi^{2} \eta\right)$ rather than in units of the magnetic diffusion time $\tau_{\eta}=D^{2} / \eta=\pi^{2}(1-\chi)^{2} \tau_{\text {dip }}$. 


\subsection{Timescales of interest}

The characteristic timescale of the Earth's magnetic field for a given spherical harmonic ( $\mathrm{SH}$ ) degree $n$ can be described by the correlation time

$$
\tau_{n}=\sqrt{\frac{\left\langle\sum_{m=0}^{n}\left[\left(g_{n}^{m}\right)^{2}+\left(h_{n}^{m}\right)^{2}\right]\right\rangle}{\left\langle\sum_{m=0}^{n}\left[\left(\dot{g}_{n}^{m}\right)^{2}+\left(\dot{h}_{n}^{m}\right)^{2}\right]\right\rangle}},
$$

where $\left\{g_{n}^{m}, h_{n}^{m}\right\}$ and $\left\{\dot{g}_{n}^{m}, \dot{h}_{n}^{m}\right\}$ are respectively the Gauss coefficients and their time derivatives of SH degree $n$ and order $m$; the angle brackets denote time averaging (e.g., Hulot \& Le Mouël 1994; Christensen \& Tilgner 2004). It provides a statistical measure of how long it would take for the observed field at a given spherical harmonic degree $n$ to be completely renewed. This timescale decreases with increasing spherical harmonic degree (i.e. the smaller the length scale, the shorter the correlation time), and such a decrease was shown from both numerical geodynamo simulations and geomagnetic field models to be statistically compatible with an inverse linear law, $\tau_{n}=\tau_{\mathrm{SV}} / n(n \geq 2)$, where $\tau_{\mathrm{SV}}$ is known as the secular-variation constant (e.g., Lhuillier et al. 2011b).

Following Lhuillier et al. (2013), we define the concepts of (successful) reversal when the dipole changes its polarity on a longterm basis, failed reversal (or grand excursion) when the dipole displays a low intensity with its pole temporarily crossing the equator, stable low intensity event (or small excursion) when the dipole displays a low intensity without its pole crossing the equator. We define therefrom the chron length between two consecutive successful reversals and the segment length between two consecutive successful or failed reversals. These events are detected according to the b-filtered algorithm described in Lhuillier et al. (2013), which filters out the segments that are shorter than the shortest duration of reversal. The average chron (resp. segment) length is hereinafter denoted by $\mu_{\mathrm{chr}}$ (resp. $\mu_{\mathrm{seg}}$ ).

\subsection{Simulations}

We investigate 56 dynamo cases obtained with the numerical code PARODY-JA (Dormy et al. 1998; Aubert et al. 2008). All simulations operate with fixed parameters $\operatorname{Pr}=1, \operatorname{Pm}=20$ and $E^{\star}=$ $2.75 \times 10^{-3}$, whereas the aspect ratio $\chi$ and the Rayleigh number $R a^{\star}$ are varied (Table 1). For each $\chi$, we explore the range of $R a^{\star}$ yielding dipolar and reversing dynamos in the vicinity of the reversal threshold $R a_{\mathrm{rcrit}}^{\star}$. Note that the value $E^{\star}=2.75 \times 10^{-3}$ corresponds to $E=6.50 \times 10^{-3}$ for the present-day aspect ratio $\chi=0.35$. This family of dynamos was extensively studied for this latter aspect ratio (e.g., Olson 2007; Driscoll \& Olson 2009; Olson et al. 2010, 2013; Lhuillier et al. 2013; Lhuillier \& Gilder 2013; Olson \& Amit 2014). All simulations operate with a lateral resolution of $44 \mathrm{SH}$ degrees, which ensures that the ratio between the minimum and maximum of the spatial power spectra exceeds 1000 for the velocity and magnetic fields. The simulations are integrated over hundreds to thousands of dipole decay times $\tau_{\text {dip }}$ in order to enable a statistical analysis of chron and segment durations.

\section{RESULTS}

To give an overview of the explored parameter space, Figure 1a shows the modified magnetic Reynolds number $R m^{\star}$ as a function of the modified magnetic Ekman number $E_{\eta}^{\star}=E^{\star} / P m$. The biplot $\left(R m, E_{\eta}\right)$, which quantifies the ratios between the timescales

of magnetic advection, magnetic diffusion and rotation, has been shown to be a convenient way to identify dynamo cases likely to be "Earth-like", despite not being run with fully appropriate dimensionless numbers, when assuming a present-day aspect ratio (Christensen et al. (2010), see also Davies \& Constable (2014)). Using the biplot $\left(R m^{\star}, E_{\eta}^{\star}\right)$ allows us to generalise this representation to also plot the parameter location of dynamos with other aspect ratios $\chi$, using dimensionless parameters rendered independent of this ratio. Our simulations largely intersect the "Earth-like" wedge-shaped zone defined by Christensen et al. (2010) for fixedflux boundary conditions, with simulations for $0.26 \leq \chi \leq 0.35$ at the lower bound and simulations for $\chi=0.10$ at the upper bound. Note, however, that such a comparison is only indicative since the expected morphology of the Earth's magnetic field for aspect ratios $\chi \neq 0.35$ is poorly known.

To pursue the survey of the parameter space, Figure 1b shows the dimensionless magnetic field (Lehnert number $\mathrm{Le}$ ) normalised by the square root of the dynamo efficiency $f_{\mathrm{ohm}}$ as a function of the convective power density $p$. Our dynamo cases fall inside the $3 \sigma$-envelop (99.7 per cent of the cases) of the diffusion-free scaling law determined by Aubert et al. (2009). In possible consistency with diffusion-free scaling laws (Christensen \& Aubert 2006; Aubert et al. 2009), Le $/ \sqrt{f_{\text {ohm }}}$ tends to increase with $p$. Nevertheless, we observe a large variability in the slope of the dependency as a function of $\chi$ (Figure 1c), which may reflect the necessity of including viscosity for more precise scaling laws (e.g., King \& Buffett 2013; Stelzer \& Jackson 2013). Associated with a lower dipolarity, the cases for $\chi=0.20$ and $\chi=0.22$ are the most distinctive with a slope two or three times shallower than the slope determined for the whole dataset.

\subsection{Scaling of the secular-variation constant and dipolarity}

Following Lhuillier et al. (2013), we first checked the behaviour of the secular-variation constant $\left(\tau_{\mathrm{SV}}\right)$ that characterises the temporal variability of the magnetic field. It had been found by Christensen \& Tilgner (2004) and Lhuillier et al. (2011a, 2013) that, for an aspect ratio of $\chi=0.35$, the inverse of $\tau_{\mathrm{SV}}$ normalised by the magnetic diffusion time $\tau_{\eta}$ linearly increases with the magnetic Reynolds number $R m$. A similar common trend can be seen for most aspect ratios, when plotting the inverse of $\tau_{\mathrm{SV}}$ normalised by the dipole decay time $\tau_{\text {dip }}$ as a function of $\mathrm{Rm}^{\star}$, both independent of $\chi$ (Figure 1d). Worth noting are the exceptions found for $\chi=0.22$, and to a lesser extent for $\chi=0.20$ and $\chi=0.24$.

We next checked the behaviour of the dipolarity $f_{\text {dip }}$ that characterises the geometry of the magnetic field. Its highest values $\left(0.55 \leq f_{\text {dip }} \leq 0.60\right)$ are found for $\chi=0.35$ and $\chi=0.40$, whereas its lowest values $\left(0.35 \leq f_{\text {dip }} \leq 0.40\right)$ are found for $\chi=0.20$ and $\chi=0.22$. It suggests that the cases for $\chi=0.20$ and $\chi=0.22$ are close to the transition between the dipole-dominated and multipolar regimes, usually defined at $f_{\text {dip }}=0.35$ (e.g., Christensen \& Aubert 2006). Figure 1e shows $f_{\text {dip }}$ as a function of $R o E^{1 / 3}$, as suggested by Oruba \& Dormy (2014) to better characterise this transition. Note, incidentally, that a very similar diagram is obtained when plotting $f_{\text {dip }}$ as a function of $R o^{\star} E^{\star 1 / 3}$, but we prefer here to use the same plotting convention as in Oruba \& Dormy (2014) to ease comparisons with other studies. In agreement with Oruba \& Dormy (2014), $f_{\text {dip }}$ tends to decrease with $R o E^{1 / 3}$. Exceptions are again found for $\chi=0.22$ and $\chi=0.24$. For the present dataset, Figure 1f shows that $f_{\text {dip }}$ actually better scales with the dynamo efficiency $f_{\mathrm{ohm}}$. The linear relationship 


\section{$4 \quad$ F. Lhuillier et al.}

5

Table 1. Key parameters of the investigated models: aspect ratio $\chi$, Ekman number $E$, Rayleigh number $R a$, modified Rayleigh number $R a^{\star}$, Rossby number $R o$, local Rossby number $R o \ell$, Lehnert number $L e$, magnetic Reynolds number $R m$, modified magnetic Reynolds number $R m^{\star}$, convective power density $p$, secular-variation constant $\tau_{\mathrm{SV}} / \tau_{\text {dip }}$, dipolarity $f_{\text {dip }}$, dynamo efficiency $f_{\mathrm{ohm}}$, run duration $T_{\text {run }} / \tau_{\text {dip }}$, number of chrons $N_{\text {chr }}$, mean chron duration $\mu_{\mathrm{chr}} / \tau_{\mathrm{dip}}$. See Section 2.1 for details. The bold rows correspond to the dynamo cases shown in Figure 2.

\begin{tabular}{|c|c|c|c|c|c|c|c|c|c|c|c|c|c|c|c|}
\hline$\chi$ & $E$ & $R a$ & $R a^{\star}$ & $R o$ & $R o_{\ell}$ & $L e$ & $R m$ & $R m^{\star}$ & $p$ & $\frac{\tau_{\mathrm{SV}}}{\tau_{\mathrm{dip}}}$ & $f_{\text {dip }}$ & $f_{\mathrm{ohm}}$ & $\frac{T_{\text {run }}}{\tau_{\text {dip }}}$ & $N_{\mathrm{chr}}$ & $\frac{\mu_{\mathrm{chr}}}{\tau_{\mathrm{dip}}}$ \\
\hline & $\cdot 10^{-3}$ & $\cdot 10^{-3}$ & $\cdot 10^{-3}$ & $\cdot 10^{-2}$ & $\cdot 10^{-2}$ & $\cdot 10^{-2}$ & & & $\cdot 10^{+3}$ & $\cdot 10^{-2}$ & & & $\cdot 10^{+3}$ & & \\
\hline 0.10 & 3.40 & 3.47 & 3.12 & 5.10 & 4.81 & 7.33 & 300 & 333 & 20.70 & 7.22 & 0.44 & 0.22 & 0.59 & & \\
\hline 0.10 & 3.40 & 3.70 & 3.33 & 5.33 & 4.99 & 7.63 & 313 & 348 & 23.00 & 7.01 & 0.43 & 0.22 & 1.37 & 21 & 64.4 \\
\hline 0.10 & 3.40 & 3.93 & 3.54 & 5.55 & 5.12 & 7.99 & 326 & 363 & 25.40 & 6.81 & 0.43 & 0.22 & 1.60 & 30 & 52.5 \\
\hline 0.10 & 3.40 & 4.16 & 3.75 & 5.78 & 5.36 & 8.05 & 340 & 378 & 27.50 & 6.63 & 0.42 & 0.22 & 3.05 & 79 & 37.7 \\
\hline 0.10 & 3.40 & 4.39 & 3.95 & 6.00 & 5.53 & 8.24 & 353 & 392 & 29.70 & 6.47 & 0.41 & 0.22 & 2.46 & 84 & 28.3 \\
\hline 0.10 & 3.40 & 4.62 & 4.16 & 6.21 & 5.73 & 8.40 & 365 & 406 & 32.10 & 6.33 & 0.41 & 0.22 & 1.52 & 62 & 23.6 \\
\hline 0.10 & 3.40 & 4.86 & 4.37 & 6.39 & 5.99 & 8.61 & 376 & 418 & 34.30 & 6.19 & 0.40 & 0.22 & 1.71 & 78 & 21.0 \\
\hline 0.10 & 3.40 & 5.09 & 4.58 & 6.61 & 6.08 & 8.54 & 389 & 432 & 36.40 & 6.07 & 0.39 & 0.21 & 1.47 & 88 & 15.7 \\
\hline 0.18 & 4.08 & 3.33 & 2.73 & 4.33 & 3.56 & 6.74 & 212 & 259 & 7.73 & 7.57 & 0.49 & 0.22 & 0.86 & & \\
\hline 0.18 & 4.08 & 3.50 & 2.87 & 4.54 & 3.87 & 6.65 & 222 & 271 & 8.48 & 7.49 & 0.48 & 0.20 & 6.88 & 51 & 131.5 \\
\hline 0.18 & 4.08 & 3.66 & 3.00 & 4.74 & 4.16 & 6.57 & 232 & 283 & 9.28 & 7.44 & 0.46 & 0.19 & 5.58 & 71 & 76.1 \\
\hline 0.18 & 4.08 & 3.83 & 3.14 & 4.94 & 4.44 & 6.49 & 242 & 295 & 10.10 & 7.38 & 0.45 & 0.18 & 5.22 & 95 & 52.3 \\
\hline 0.18 & 4.08 & 4.00 & 3.28 & 5.14 & 4.71 & 6.34 & 252 & 307 & 10.90 & 7.29 & 0.43 & 0.17 & 5.13 & 149 & 32.0 \\
\hline 0.20 & 4.29 & 3.50 & 2.80 & 4.27 & 3.68 & 6.25 & 199 & 249 & 6.46 & 7.75 & 0.47 & 0.20 & 0.82 & 7 & 112.6 \\
\hline 0.20 & 4.29 & 3.68 & 2.94 & 4.53 & 4.03 & 6.12 & 211 & 264 & 7.19 & 7.61 & 0.45 & 0.18 & 0.81 & 15 & 50.9 \\
\hline 0.20 & 4.29 & 3.86 & 3.09 & 4.91 & 4.54 & 5.49 & 229 & 286 & 7.97 & 7.25 & 0.41 & 0.13 & 0.78 & 26 & 27.6 \\
\hline 0.20 & 4.29 & 4.05 & 3.24 & 5.11 & 4.80 & 5.02 & 238 & 298 & 8.75 & 7.40 & 0.39 & 0.12 & 0.80 & 67 & 11.1 \\
\hline 0.20 & 4.29 & 4.23 & 3.39 & 5.24 & 4.96 & 5.04 & 244 & 305 & 9.48 & 7.55 & 0.39 & 0.12 & 0.75 & 78 & 8.5 \\
\hline 0.22 & 4.51 & 3.66 & 2.86 & 4.59 & 4.30 & 3.59 & 203 & 261 & 5.55 & 7.21 & 0.35 & 0.07 & 3.83 & 40 & 92.8 \\
\hline 0.22 & 4.51 & 3.76 & 2.94 & 4.67 & 4.37 & 3.75 & 207 & 266 & 5.86 & 7.35 & 0.36 & 0.07 & 2.06 & 52 & 37.3 \\
\hline 0.22 & 4.51 & 3.86 & 3.01 & 4.73 & 4.43 & 4.09 & 210 & 269 & 6.18 & 7.68 & 0.38 & 0.09 & 3.75 & 183 & 19.6 \\
\hline 0.22 & 4.51 & 3.97 & 3.09 & 4.82 & 4.54 & 4.11 & 214 & 274 & 6.52 & 7.72 & 0.38 & 0.09 & 2.30 & 162 & 13.3 \\
\hline 0.22 & 4.51 & 4.07 & 3.17 & 4.90 & 4.64 & 4.24 & 217 & 279 & 6.85 & 7.81 & 0.39 & 0.09 & 3.74 & 417 & 8.7 \\
\hline 0.24 & 4.75 & 2.48 & 1.89 & 2.68 & 1.85 & 3.87 & 113 & 149 & 1.56 & 11.23 & 0.45 & 0.15 & 0.84 & & \\
\hline 0.24 & 4.75 & 2.71 & 2.06 & 2.88 & 1.87 & 4.32 & 121 & 160 & 1.83 & 9.42 & 0.42 & 0.16 & 0.88 & & \\
\hline 0.24 & 4.75 & 2.93 & 2.23 & 3.10 & 2.52 & 5.41 & 131 & 172 & 2.52 & 10.41 & 0.51 & 0.22 & 0.87 & & \\
\hline 0.24 & 4.75 & 3.16 & 2.40 & 3.41 & 2.94 & 5.37 & 144 & 189 & 2.98 & 9.78 & 0.50 & 0.20 & 0.95 & 3 & 317.6 \\
\hline 0.26 & 5.02 & 2.77 & 2.05 & 2.77 & 2.61 & 5.32 & 111 & 149 & 1.88 & 18.45 & 0.50 & 0.25 & 2.01 & 40 & 47.7 \\
\hline 0.26 & 5.02 & 3.02 & 2.24 & 2.92 & 2.59 & 6.01 & 116 & 157 & 2.14 & 12.67 & 0.53 & 0.28 & 7.14 & 17 & 419.0 \\
\hline 0.26 & 5.02 & 3.28 & 2.42 & 3.15 & 2.93 & 6.58 & 126 & 170 & 2.71 & 11.28 & 0.53 & 0.30 & 7.14 & 61 & 115.2 \\
\hline 0.26 & 5.02 & 3.40 & 2.52 & 3.30 & 3.10 & 6.31 & 132 & 178 & 2.89 & 11.14 & 0.52 & 0.27 & 7.00 & 121 & 56.6 \\
\hline 0.26 & 5.02 & 3.53 & 2.61 & 3.48 & 3.26 & 5.86 & 139 & 187 & 3.02 & 10.73 & 0.50 & 0.23 & 7.37 & 183 & 39.2 \\
\hline 0.30 & 5.60 & 3.45 & 2.41 & 2.89 & 2.76 & 6.88 & 103 & 147 & 1.70 & 15.61 & 0.54 & 0.34 & 0.67 & 15 & 42.8 \\
\hline 0.30 & 5.60 & 3.76 & 2.63 & 3.13 & 3.01 & 7.52 & 112 & 159 & 2.18 & 12.78 & 0.54 & 0.35 & 3.93 & 114 & 32.7 \\
\hline 0.30 & 5.60 & 4.08 & 2.85 & 3.39 & 3.32 & 7.20 & 121 & 173 & 2.53 & 11.92 & 0.52 & 0.31 & 3.66 & 212 & 15.9 \\
\hline 0.30 & 5.60 & 4.39 & 3.07 & 3.68 & 3.66 & 6.90 & 131 & 188 & 2.87 & 10.90 & 0.51 & 0.27 & 3.97 & 364 & 9.5 \\
\hline 0.30 & 5.60 & 4.70 & 3.29 & 3.97 & 3.98 & 6.67 & 142 & 202 & 3.21 & 9.73 & 0.49 & 0.25 & 3.71 & 394 & 8.1 \\
\hline 0.30 & 5.60 & 5.02 & 3.51 & 4.18 & 4.23 & 6.84 & 149 & 213 & 3.57 & 9.13 & 0.50 & 0.24 & 1.17 & 108 & 9.4 \\
\hline 0.35 & 6.50 & 4.90 & 3.19 & 3.38 & 3.33 & 8.23 & 104 & 160 & 1.62 & 13.02 & 0.58 & 0.36 & 3.08 & 36 & 80.3 \\
\hline 0.35 & 6.50 & 5.07 & 3.30 & 3.52 & 3.55 & 7.97 & 108 & 167 & 1.74 & 11.42 & 0.57 & 0.33 & 9.14 & 221 & 38.1 \\
\hline 0.35 & 6.50 & 5.28 & 3.43 & 3.67 & 3.79 & 7.78 & 113 & 174 & 1.89 & 12.05 & 0.55 & 0.31 & 5.81 & 218 & 23.9 \\
\hline 0.35 & 6.50 & 5.49 & 3.57 & 3.78 & 4.00 & 7.73 & 116 & 179 & 2.03 & 11.61 & 0.55 & 0.30 & 8.12 & 350 & 20.7 \\
\hline 0.35 & 6.50 & 5.70 & 3.71 & 3.91 & 4.20 & 7.58 & 120 & 185 & 2.16 & 11.21 & 0.54 & 0.28 & 5.10 & 229 & 19.4 \\
\hline 0.35 & 6.50 & 5.92 & 3.84 & 4.04 & 4.39 & 7.44 & 124 & 191 & 2.29 & 10.71 & 0.53 & 0.27 & 6.66 & 328 & 17.1 \\
\hline 0.35 & 6.50 & 6.34 & 4.12 & 4.33 & 4.74 & 7.21 & 133 & 205 & 2.58 & 9.66 & 0.50 & 0.24 & 6.57 & 237 & 22.2 \\
\hline 0.35 & 6.50 & 6.76 & 4.39 & 4.51 & 5.08 & 7.42 & 139 & 213 & 2.86 & 9.38 & 0.51 & 0.23 & 5.12 & 168 & 24.2 \\
\hline 0.40 & 7.63 & 7.57 & 4.54 & 4.33 & 4.84 & 8.20 & 113 & 189 & 1.60 & 11.09 & 0.59 & 0.30 & 6.73 & 20 & 331.9 \\
\hline 0.40 & 7.63 & 8.15 & 4.89 & 4.49 & 5.25 & 7.90 & 118 & 196 & 1.78 & 10.72 & 0.58 & 0.26 & 6.75 & 66 & 96.9 \\
\hline 0.40 & 7.63 & 8.73 & 5.24 & 4.70 & 5.69 & 7.59 & 123 & 205 & 1.98 & 10.28 & 0.57 & 0.23 & 6.68 & 112 & 53.9 \\
\hline 0.40 & 7.63 & 9.31 & 5.59 & 4.90 & 6.14 & 7.51 & 128 & 214 & 2.25 & 10.06 & 0.55 & 0.21 & 5.87 & 174 & 28.9 \\
\hline 0.40 & 7.63 & 9.90 & 5.94 & 5.14 & 6.57 & 7.55 & 135 & 224 & 2.55 & 9.93 & 0.51 & 0.20 & 4.49 & 280 & 13.1 \\
\hline 0.44 & 8.76 & 9.98 & 5.59 & 5.04 & 5.95 & 8.02 & 115 & 205 & 1.45 & 11.04 & 0.53 & 0.25 & 11.55 & 52 & 217.7 \\
\hline 0.44 & 8.76 & 10.74 & 6.02 & 5.06 & 6.46 & 7.63 & 116 & 206 & 1.55 & 10.73 & 0.54 & 0.22 & 9.04 & 99 & 85.4 \\
\hline 0.44 & 8.76 & 11.51 & 6.45 & 5.17 & 7.01 & 7.30 & 118 & 211 & 1.73 & 10.14 & 0.50 & 0.19 & 5.67 & 125 & 37.9 \\
\hline 0.44 & 8.76 & 12.28 & 6.88 & 5.30 & 7.53 & 7.38 & 121 & 216 & 1.94 & 9.93 & 0.47 & 0.18 & 3.08 & 181 & 13.6 \\
\hline 0.44 & 8.76 & 13.05 & 7.31 & 5.49 & 7.98 & 7.66 & 125 & 224 & 2.17 & 10.01 & 0.45 & 0.18 & 1.41 & 154 & 7.4 \\
\hline
\end{tabular}




\subsection{Impact of inner-core size on the dipole field behaviour}

The overall behaviour of the dipolar field produced by the dynamos can be characterised with the help of the dipole-phase-space (DPS) diagrams defined in Lhuillier et al. (2013). Key examples of such diagrams are presented in Figure 2a-f for simulations operating, for each aspect ratio $\chi$, approximately $13 \%$ above the reversal thresh-
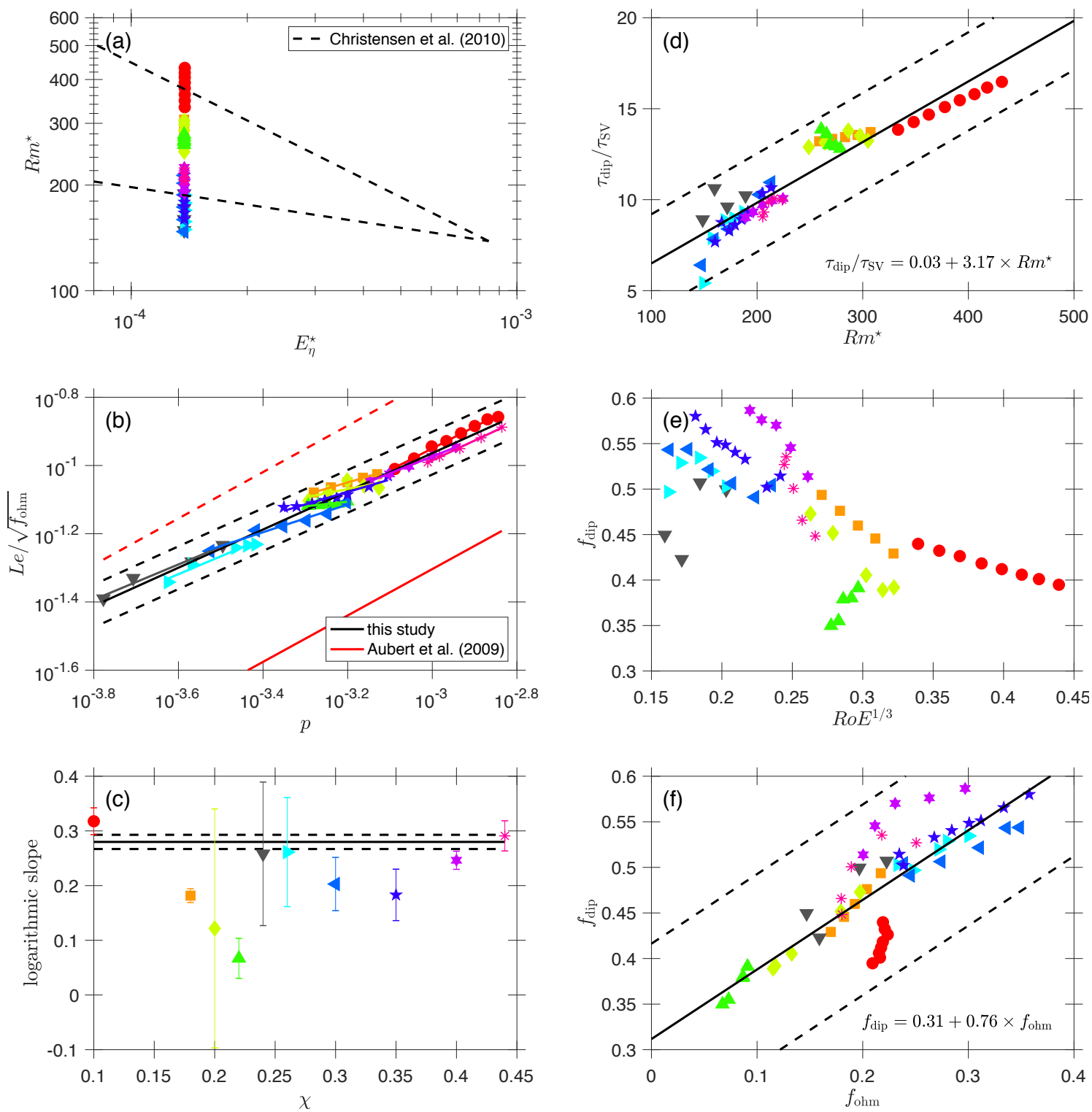

Figure 1. Parameter space of the investigated models. Panel (a): Location of the dynamo cases in terms of the modified magnetic Reynolds number $R m^{\star}$ and the modified magnetic Ekman number $E_{\eta}^{\star}=E^{\star} / P m$ with respect to the "Earth-like" wedge-shaped zone (dashed lines) defined by Christensen et al. (2010) for fixed-flux boundary conditions. Panel (b): Lehnert number $L e$ (dimensionless magnetic field) normalised by the square root of the dynamo efficiency $f_{\mathrm{ohm}}$ as a function of convective power density $p$. Panel (c): Slope of $\log \left(L e / \sqrt{f_{\mathrm{ohm}}}\right)$ vs $\log (p)$ as a function of aspect ratio $\chi$. Panel (d): Inverse of the secular-variation constant $\tau_{\mathrm{SV}} / \tau_{\mathrm{dip}}$ as a function of magnetic Reynolds number $R m^{\star}$. Panel (e): Dipolarity $f_{\text {dip }}$ as a function of the parameter $R o E^{1 / 3}$ defined by Oruba \& Dormy (2014). Panel (f): Dipolarity $f_{\text {dip }}$ as a function of $f_{\text {ohm }}$. Best-fitting laws are provided with their $3 \sigma$ dispersion lines (e.g., Aubert et al. 2009).

$f_{\text {dip }}=0.31+0.76 \times f_{\text {ohm }}$ suggests a minimal value $f_{\text {dip }}=0.31$ for this family of dynamos. old $R a_{\mathrm{rcrit}}^{\star}$. In addition to their two distinct stable modes of opposite polarity, these dynamos can be discriminated by the occurrence and size of a central unstable mode associated with the transitional times. Useful complementary information can also be recovered using histograms of the axial dipole moment (ADM, Figure 2g-1). This reveals that for such dynamos, changing the aspect ratio $\chi$ can have a strong influence.

For $\chi=0.10$, the DPS diagram shows that the stable modes are well separated and the central unstable mode almost nonexistent, indicating that the polarity transitions occur with little time 


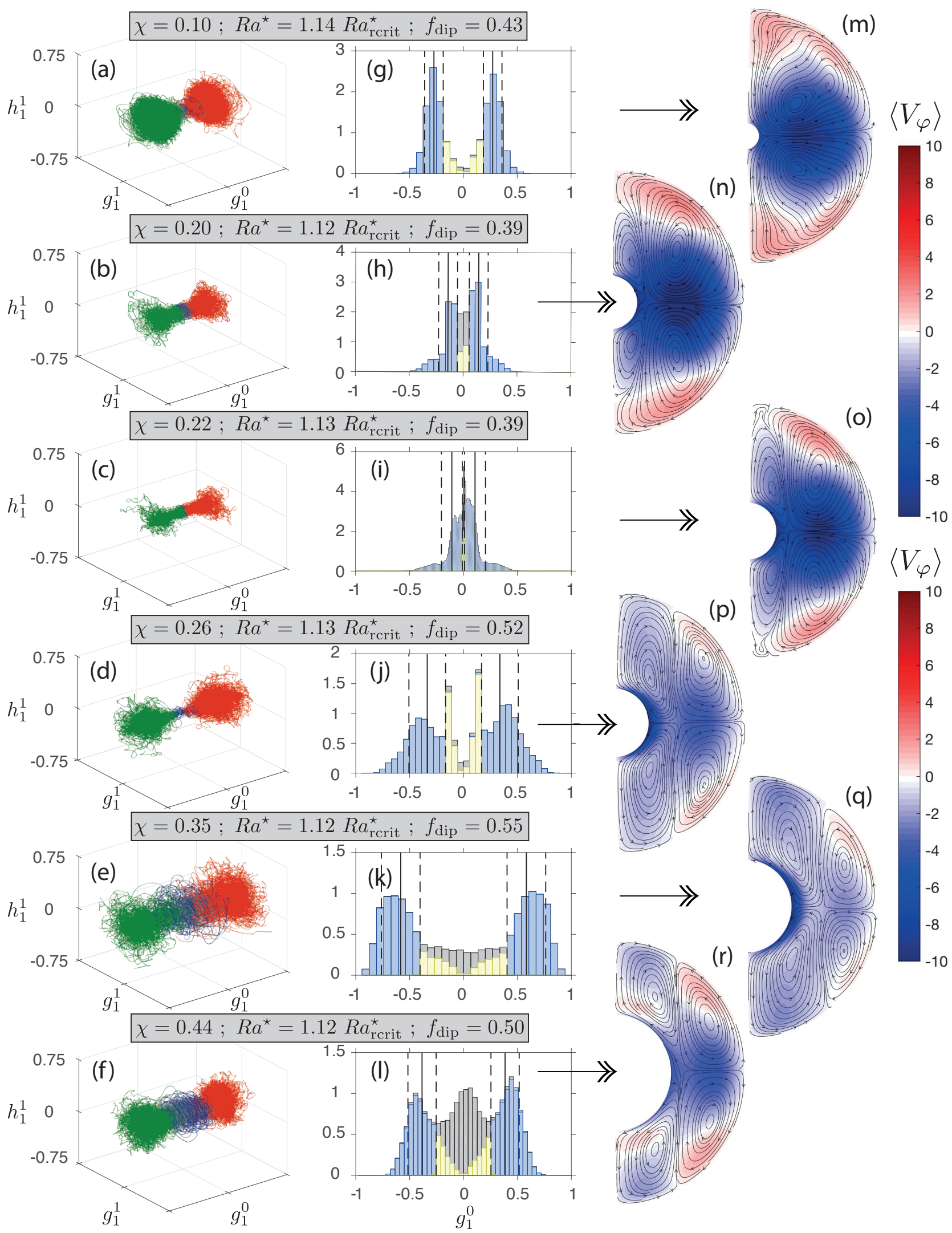

Figure 2. Dipole behaviour and flow pattern for various aspect ratios $\chi$, where the Gauss coefficients $\left(g_{0}^{1}, g_{1}^{1}, h_{1}^{1}\right)$ are expressed at the CMB in units of $(\rho \mu \eta \Omega)^{1 / 2}$ with the same plotting conventions as in Lhuillier et al. (2013). Panels (a)-(f): Dipole-phase-space diagrams. Red (resp. green) colours when the dynamo is evolving in a normal (resp. reverse) polarity segment. Blue (resp. grey) colours when the dynamo is experiencing a reversal (resp. a grand excursion). Panels (g)-(l): Histograms of the axial dipole moments. The vertical lines correspond to the averaged values $( \pm \sigma)$ within the polarity segments. We highlight in light blue contributions within positive or negative polarity segments, in grey contributions at times of reversals or failed reversals, and in yellow the contributions of the small excursions. Panels (m)-(r): Meridional cuts of the flow. The coloured surfaces (westward in blue, eastward in red) account for the azimuthal velocity field, the black arrows for the motion in the meridional plane. Both quantities are averaged over time and longitude. 
spent at low dipole field intensity. The ADM distribution also shows that, at times of stable polarity, the field is relatively strong. Increasing the aspect ratio to $\chi=0.20$ leads to a noticeable change of the dipole behaviour. Stable modes become less marked and the central unstable mode much more significant, showing that polarity transitions occur with more time spent at low dipole field intensity. This is also confirmed by the ADM distribution, which further show that, at times of stable polarity, the field is substantially weaker for this aspect ratio. These characteristics show up even more strongly when the aspect ratio reaches $\chi=0.22$ and the field becomes even weaker. In that case, limits between the central unstable mode and the two stable modes become difficult to distinguish, even though each stable mode can well be identified (the weak dipole remaining mainly axial). Increasing the aspect ratio to $\chi=0.26$ leads to a clear change of trend in the behaviour of the dynamos. A central unstable mode with weak dipole moment is often experienced by the dynamo, while the two stable polarity modes are now again associated with stronger dipole field moments. For $\chi=0.35$, the central unstable mode grows further and the two stable polarity modes now display strong dipole moments. Increasing the aspect ratio to $\chi=0.44$ leads to a more prominent central unstable mode, together with a slight decrease in the overall dipole moment.

The previous analysis thus reveals two key transitions in the dipole field behaviour when the size of the inner core increases: one when the aspect ratio reaches $\chi=0.20$; the other when it reaches $\chi=0.26$. To document what may cause these changes, we plotted meridional cuts of the dynamo flow averaged over time and longitude (Figure $2 \mathrm{~m}-\mathrm{r}$ ). These cuts show that, as $\chi$ increases, changes in the average flows mainly occur within the so-called tangent cylinder (the cylinder parallel to the rotation axis and tangent to the inner core). For a small enough inner core $(\chi=0.10)$, a downwelling flow from the poles to the inner core is found in both hemispheres. As $\chi$ increases, the flow closest to the inner core starts moving outwards towards the poles, splitting the flow within the tangent cylinder into two cells in each hemisphere. For larger aspect ratios beyond $\chi=0.26$, this downwelling is no longer there and the average flow within the tangent cylinder only consists of one upwelling cell in each hemisphere. Although we could not formally establish any causal link between these two major changes, changes in the dipole field behaviour as the aspect ratio increases appear to be closely related to concomitant changes in the dynamo flows within the tangent cylinder, the range between $\chi=0.20$ and $\chi=0.22$ marking a transition between a "small inner-core" regime and a "large inner-core" regime. It is also worth recalling that the dynamo efficiency $f_{\text {ohm }}$ tends to be weaker during the transition between these two regimes, leading to a less dipolar field and that the secular variation constant $\tau_{\mathrm{SV}}$ then also reacts differently when increasing $R m^{\star}$ (Figure 1).

\subsection{Scaling of the reversal frequency}

We now focus on the frequency of reversals that depends both on the vigour of the convection and the aspect ratio of the dynamos investigated here. For the present-day geometry $(\chi=0.35)$, Lhuillier et al. (2013) found that the dimensionless reversal frequency defined by $\tau_{\eta} / \mu_{\mathrm{chr}}$ is, as a first approximation, a linear function of the magnetic Reynolds number $R m$. Using the revised dimensional reversal frequency defined by $\tau_{\mathrm{dip}} / \mu_{\mathrm{chr}}$ to remain independent of the aspect ratio, we found that $\tau_{\mathrm{dip}} / \mu_{\mathrm{chr}}$ is indeed close to a linear function of $R m^{\star}$ for any aspect ratio (Figure 3a). Alternatively, Olson \& Amit (2014) proposed that $\tau_{\mathrm{dip}} / \mu_{\mathrm{chr}}$ is, as a first approximation, a linear function of the local Rossby number $R o_{\ell}$. This, however, appears to be less compatible with the present dataset (Figure $3 \mathrm{~b}$ ). We also tried to represent $\tau_{\mathrm{dip}} / \mu_{\mathrm{chr}}$ as a function of the modified Rayleigh number $R a^{\star}$ (Figure $3 \mathrm{c}$ ) or the convective power density $p$ (Figure $3 \mathrm{~d}$ ). As $p$ was found to scale $\tau_{\text {dip }} / \mu_{\text {chr }}$ slightly better than the other parameters (or at least, as efficiently as $R m^{\star}$ ) and is easier to interpret with respect to the evolution of the geodynamo, we decided to pursue our analysis solely with this parameter. Considering a linear dependency between $\tau_{\text {dip }} / \mu_{\text {chr }}$ and $p$ is of course a strong assumption. Nevertheless, given the uncertainties on the determination of $\tau_{\mathrm{dip}} / \mu_{\mathrm{chr}}$ and the limited number of dynamo cases for each aspect ratio, it is difficult to produce a finer description of the dependency for the present dataset. Also note that the linear fits were restricted to the dynamo cases for which the reversal frequency could be unambiguously determined. In particular, we excluded from our analysis some chaotic dynamos at high $R a$ characterised by a mechanical decrease of the reversal frequency due to the filtering of the shortest chrons.

Several important conclusions can be drawn from Figure 3d, where fits of the form $\tau_{\text {dip }} / \mu_{\mathrm{chr}}=a \times\left(p-p_{\text {rcrit }}\right)$ were computed for each aspect ratio. First, the rate $a$ at which the reversal frequency varies with $p$ appears to slowly increase by approximately a factor 2 from $\chi=0.10$ to $\chi=0.45$ (Figure $3 \mathrm{e}$ ). Of particular interest, though, is that the dynamo cases for $\chi=0.20$ and $\chi=0.22$ produce rates exceeding the trend by a factor 3 to 4 . Second, the critical value $p_{\text {rcrit }}$ above which reversals start occurring tends to decrease by approximately a factor 3 from $\chi=0.10$ to $\chi=0.26$, then recovers by approximately a factor 4 from $\chi=0.26$ to 0.44 (Figure 3f). Once again, the dynamo cases for $\chi=0.20$ and $\chi=0.22$ slightly depart from the parabolic trend. From the point of view of reversal frequency, this shows that a specific behaviour can also be identified for dynamos in the transition between the previously identified "small inner-core" and "large inner-core" regimes (Section 3.2). These dynamos are the only ones characterised by both a high value of $p_{\text {rcrit }}$ needed to produce reversals and a high ability to produce frequent reversals when $p$ increases beyond $p_{\text {rcrit }}$. Note, finally, that for $\chi=0.24$, the range of $p$ over which we successfully found dynamos produced reversals for only one case (out of 4), which prevented us from assessing the dependency of $\tau_{\text {dip }} / \mu_{\text {chr }}$ on $p$.

\section{DISCUSSION}

In this systematic study of 56 chemically-driven dynamo cases, we varied the inner-core size (i.e. the aspect ratio $\chi$ ) and the forcing parameter of the convection (i.e. the modified Rayleigh number $R a^{\star}$ ) while maintaining a balance between the rotational and diffusive forces. For this purpose, we kept the modified Ekman number $E^{\star}$ constant, rather than the conventional Ekman number $E$ that depends on $\chi$. For these dynamos, we found that one can define a "small inner-core" regime $(\chi \leq 0.18)$ characterised by intermediately strong and dipolar fields, and a "large inner-core" regime $(\chi \geq 0.26)$ characterised by a stronger and more dipolar field, the transition occurring between $\chi=0.20$ and $\chi=0.24$, when the field is then substantially weaker and slightly less dipolar. This evolution can be associated with a progressive change in the average dynamo flow, which evolves from polar downwelling towards the inner core when the inner core is small, to polar upwelling from the inner core to the poles when the inner core is large (Figure 2).

This change of flow pattern is consistent with that found by Landeau et al. (2017) when comparing thermo-chemical dynamos with no inner core and large inner cores. With respect to the field 


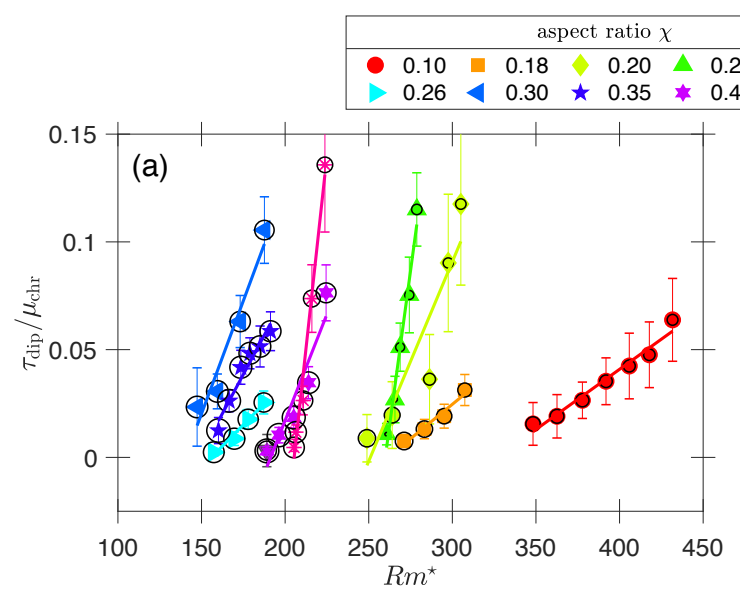

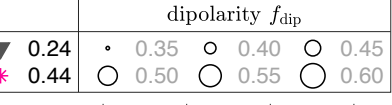
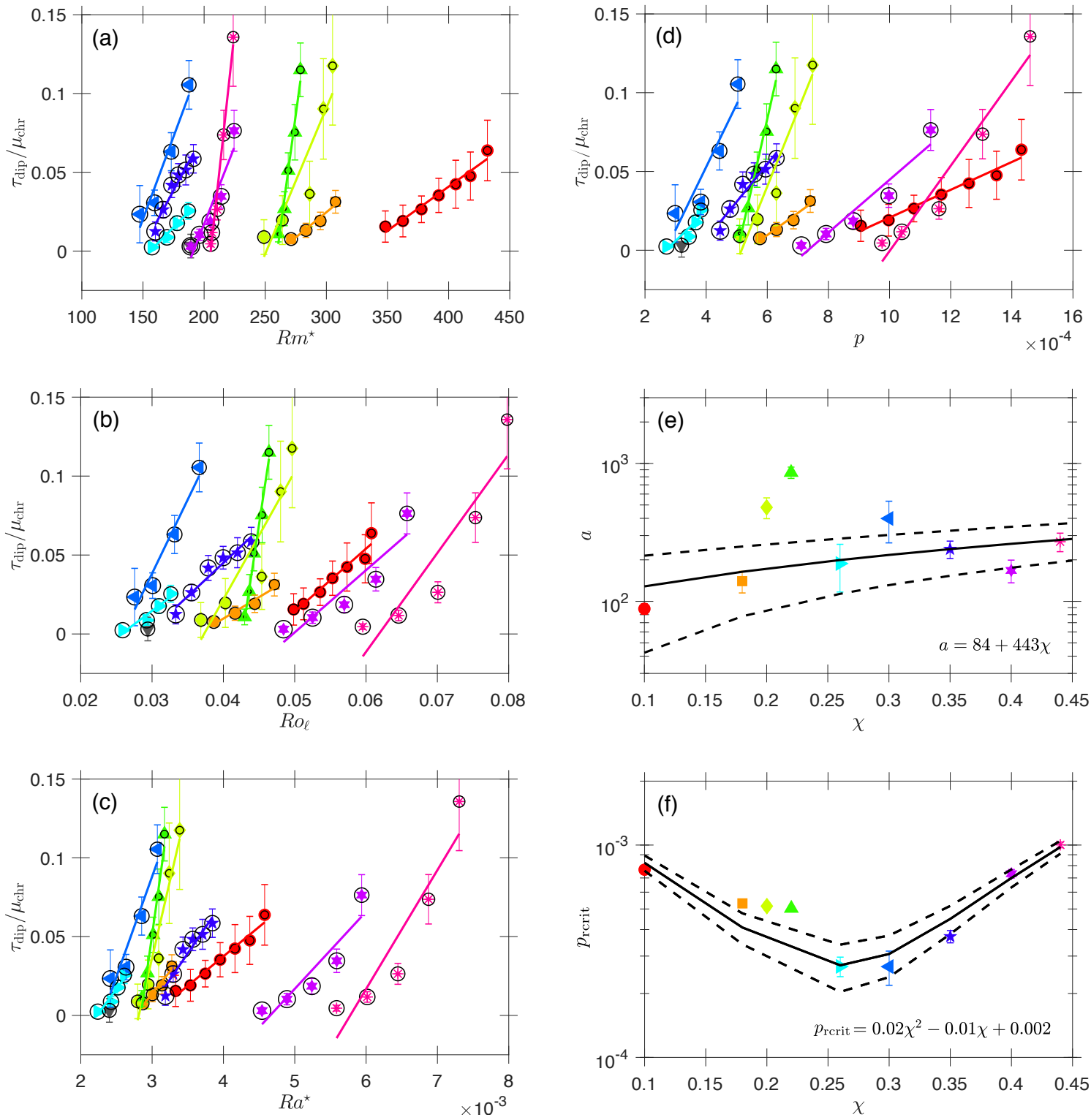

Figure 3. Evolution of the dimensionless reversal frequency $\tau_{\text {dip }} / \mu_{\text {chr }}$ (with $1 \sigma$ dispersion bars) for various aspect ratios $\chi$. Panel (a): $\tau_{\text {dip }} / \mu_{\text {chr }}$ as a function of $R m^{\star}$. Panel (b): $\tau_{\text {dip }} / \mu_{\text {chr }}$ as a function of $R o \ell$. Panel (c): $\tau_{\text {dip }} / \mu_{\text {chr }}$ as a function of $R a^{\star}$. Panel (d): $\tau_{\text {dip }} / \mu_{\text {chr }}$ as a function of $p$, with linear fits of the form $\tau_{\mathrm{dip}} / \mu_{\mathrm{chr}}=a \times\left(p-p_{\text {rcrit }}\right)$. Panels (e)-(f): slope $a$ and reversal threshold $p_{\text {rcrit }}$ as a function of $\chi$ (with $1 \sigma$ dispersion bars).

evolution, however, Landeau et al. (2017) found a general tendency for the intensity to become weaker as the inner core size increases, in contradiction with our findings. In contrast, Heimpel et al. (2005) found a trend in better agreement with our results when investigating a set of thermally-driven dynamos. One possible explanation for this discrepancy is that both our simulations and those of Heimpel et al. (2005) were operated with an electrically conducting inner core, whereas those of Landeau et al. (2017) assumed an electrically insulating inner core, a less realistic assumption known to have a strong impact on the presently investigated dynamos (e.g., Lhuillier et al. 2013). Unfortunately, neither Heimpel et al. (2005) nor Landeau et al. (2017) reported any cases between $\chi=0.20$ and $\chi=0.24$, corresponding to the transition that we observe between the "small" and "large inner-core" regimes.
In accordance with previous studies (e.g., Olson 2007; Driscoll \& Olson 2009; Lhuillier et al. 2013; Olson \& Amit 2014), our dynamos display, for any aspect ratio $\chi$, a reversal frequency that increases with the vigour of convection. For sufficiently efficient dynamos $\left(f_{\text {ohm }}>0.20\right)$ the dimensionless reversal frequency scales well with $p-p_{\text {rcrit }}$, where $p_{\text {rcrit }}$ is the critical value above which reversals start occurring. For this study, we found that the critical number $p_{\text {rcrit }}$ decreases from $\chi=0.10$ to $\chi=0.22$ and increases from $\chi=26$ to $\chi=0.44$, with a possible jump to higher values observed between $\chi=0.22$ and $\chi=0.26$. In addition, and most importantly, we found that during the transition between the previously identified "small inner-core" and "large inner-core" regimes $(0.20 \leq \chi \leq 0.22)$, the sensitivity of the reversal fre- 
quency to an increase of $p$ beyond $p_{\text {rcrit }}$ is very significantly enhanced.

Recent thermal models of the Earth favour a relatively young nucleation of the inner core between $\sim 400$ and $\sim 1100 \mathrm{Ma}$ (e.g., Olson et al. 2015), with a preferred age between $\sim 600$ and $\sim 700$ Ma. (e.g., Labrosse 2015; Davies 2015; Driscoll 2016). If we use as a guideline the thermal model of Olson et al. (2013), with a nucleation starting around $700 \mathrm{Ma}$, the transition of interest would have occurred somewhere between $\sim 500 \mathrm{Ma}(\chi=0.20)$ and $\sim 350 \mathrm{Ma}(\chi=0.26)$. Is there any palaeomagnetic evidence of a transition during such a period? Some reversal hyperactivity, with a reversal frequency perhaps exceeding 20 per Myr, has been documented at the very end of the Precambrian towards $550 \mathrm{Ma}$ (Shatsillo et al. 2015; Bazhenov et al. 2016). Magnetostratigraphic data obtained for the Middle Cambrian (Pavlov \& Gallet 2001; Gallet et al. 2003; Gallet \& Pavlov 2016, and work in progress) also argue in favour of another similar unusual event about $50 \mathrm{Myr}$ later, though both studies may document a single $\sim 50$ Myr-long hyperactivity segment (Duan et al. 2018). Such a long duration or the repetition of these events, and the unusually high reversal frequency apparently achieved, suggests a situation different from that having prevailed during the Middle Jurassic. Magnetostratigraphic data obtained during the Ordovician $(\sim 485-444 \mathrm{Ma})$ also attest for the existence of a reverse superchron (the Moyero superchron, Pavlov \& Gallet 2005). The combination of such antagonist events-hyperactivity events and superchron within a $\sim$ $20 \mathrm{Myr}$ interval-is consistent with a strong sensitivity of the reversal frequency to mild fluctuations of $p$, of the type one would expect during the transition between the "small" and "large innercore" regimes. Absolute palaeointensity data over that same period of time are only partly documented, with hardly any data available during the Cambrian (e.g., Tauxe \& Yamazaki 2007). However, they hint at a low value of the field after $\sim 500 \mathrm{Ma}$, extending to the better documented Devonian ( 419-359 Ma) when the field displayed an unusually low intensity and was less dipolar (e.g., Shcherbakova et al. 2017; Hawkins et al. 2019). Starting from the Carboniferous, many more data are available and they testify for a field becoming stronger again. This is exactly what one would expect from our results for a dynamo finally reaching the "large inner-core" regime.

Such encouraging comparisons should nevertheless be taken with substantial caution. The transition we observe between "small" and "large inner-core" regimes in the highly viscous and purely chemically-driven dynamos investigated here may not be representative of the geodynamo. In addition, the exact values of $\chi$ at which the transition occurs may be sensitive to other control parameters. Finally, and most importantly, time-varying inhomogeneous thermal boundary conditions imposed by the mantle have not been considered here. Yet, imposing such time-varying inhomogeneous boundary conditions on dynamos very similar to those investigated here has been shown to also significantly affect the reversing and field properties of these dynamos (e.g., Olson et al. 2013), with the potential ability to account for most, if not all, of the properties observed in the paleomagnetic record. Distinguishing the contribution of the progressive growth of the inner core from that of timevarying inhomogeneous boundary conditions to this long-term behaviour definitely remains a challenge. What our study nevertheless suggests is that the best way to search for the specific signature of the inner core's growth in the magnetic signal could be to search for major changes that can be expected to have occurred during the transition of the dynamo from a likely transition between a "small inner-core" regime to a "large inner-core" regime.

\section{ACKNOWLEDGMENTS}

Numerical computations were performed using the High Performance Computing (HPC) facilities of the Geophysics section at LMU (Munich, Germany). We thank two anonymous reviewers for their helpful comments, as well as Gary Egbert for editorial handling. F.L. also thanks Rob Coe for his encouraging comments on the manuscript. This is IPGP contribution XXXX.

\section{REFERENCES}

Aubert, J., Aurnou, J. M., \& Wicht, J., 2008. The magnetic structure of convection-driven numerical dynamos, Geophys. J. Int., 172(3), 945956.

Aubert, J., Labrosse, S., \& Poitou, C., 2009. Modelling the palaeoevolution of the geodynamo, Geophys. J. Int., 179(3), 1414-1428.

Aubert, J., Gastine, T., \& Fournier, A., 2017. Spherical convective dynamos in the rapidly rotating asymptotic regime, J. Fluid Mech., 813, 558-593.

Bazhenov, M. L., Levashova, N. M., Meert, J. G., Golovanova, I. V., Danukalov, K. N., \& Fedorova, N. M., 2016. Late Ediacaran magnetostratigraphy of Baltica: Evidence for Magnetic Field Hyperactivity?, Earth Planet. Sci. Lett., 435, 124-135.

Biggin, A. J., Steinberger, B., Aubert, J., Suttie, N., Holme, R., Torsvik, T. H., van der Meer, D. G., \& van Hinsbergen, D. J. J., 2012. Possible links between long-term geomagnetic variations and whole-mantle convection processes, Nat. Geosci., 5(8), 526-533.

Biggin, A. J., Piispa, E. J., Pesonen, L. J., Holme, R., Paterson, G. A., Veikkolainen, T., \& Tauxe, L., 2015. Palaeomagnetic field intensity variations suggest Mesoproterozoic inner-core nucleation, Nature, 526(7572), 245-248.

Braginsky, S. \& Roberts, P. H., 1995. Equations governing convection in Earth's core and the geodynamo, Geophys. Astrophys. Fluid Dyn., 79(14), 1-97.

Christensen, U. R. \& Aubert, J., 2006. Scaling properties of convectiondriven dynamos in rotating spherical shells and application to planetary magnetic fields, Geophys. J. Int., 166(1), 97-114.

Christensen, U. R. \& Tilgner, A., 2004. Power requirement of the geodynamo from ohmic losses in numerical and laboratory dynamos, Nature, 429(6988), 169-171.

Christensen, U. R., Aubert, J., \& Hulot, G., 2010. Conditions for Earthlike geodynamo models, Earth Planet. Sci. Lett., 296(3-4), 487-496.

Coe, R. S. \& Glatzmaier, G. A., 2006. Symmetry and stability of the geomagnetic field, Geophys. Res. Lett., 33(21), 1-5.

Courtillot, V. \& Olson, P. L., 2007. Mantle plumes link magnetic superchrons to phanerozoic mass depletion events, Earth Planet. Sci. Lett., 260(3-4), 495-504.

Davies, C. J., 2015. Cooling history of Earth's core with high thermal conductivity, Phys. Earth Planet. Inter., 247, 65-79.

Davies, C. J. \& Constable, C. G., 2014. Insights from geodynamo simulations into long-term geomagnetic field behaviour, Earth Planet. Sci. Lett., 404(0), 238-249.

Dharmaraj, G. \& Stanley, S., 2012. Effect of inner core conductivity on planetary dynamo models, Phys. Earth Planet. Inter, 212-213(0), 1-9.

Dormy, E., Cardin, P., \& Jault, D., 1998. MHD flow in a slightly differentially rotating spherical shell, with conducting inner core, in a dipolar magnetic field, Earth Planet. Sci. Lett., 160(1-2), 15-30.

Driscoll, P. E., 2016. Simulating 2 Ga of geodynamo history, Geophys. Res. Lett., 43, 1-8.

Driscoll, P. E. \& Evans, D. A. D., 2016. Frequency of Proterozoic geomagnetic superchrons, Earth Planet. Sci. Lett., 437, 9-14.

Driscoll, P. E. \& Olson, P. L., 2009. Effects of buoyancy and rotation on the polarity reversal frequency of gravitationally driven numerical dynamos, Geophys. J. Int., 178(3), 1337-1350.

Driscoll, P. E. \& Olson, P. L., 2011. Superchron cycles driven by variable core heat flow, Geophys. Res. Lett., 38(9).

Duan, Z., Liu, Q., Ren, S., Li, L., Deng, X., \& Liu, J., 2018. Magnetic 
reversal frequency in the Lower Cambrian Niutitang Formation, Hunan Province, South China, Geophys. J. Int., 214(2), 1301-1312.

Gallet, Y. \& Hulot, G., 1997. Stationary and nonstationary behaviour within the geomagnetic polarity time scale, Geophys. Res. Lett., 24(15), $1875-1878$.

Gallet, Y. \& Pavlov, V. E., 2016. Three distinct reversing modes in the geodynamo, Izv., Phys. Solid Earth, 52(2), 291-296.

Gallet, Y., Pavlov, V. E., \& Courtillot, V., 2003. Magnetic reversal frequency and apparent polar wander of the Siberian platform in the earliest Palaeozoic, inferred from the Khorbusuonka river section (northeastern Siberia), Geophys. J. Int., 154(3), 829-840.

Gallet, Y., Pavlov, V. E., Halverson, G., \& Hulot, G., 2012. Toward constraining the long-term reversing behavior of the geodynamo: A new "Maya" superchron $\sim 1$ billion years ago from the magnetostratigraphy of the Kartochka Formation (southwestern Siberia), Earth Planet. Sci. Lett., 339-340, 117-126.

Glatzmaier, G. A., 2002. Geodynamo Simulations - How Realistic Are They?, Annu. Rev. Earth Planet. Sci., 30, 237-257.

Glatzmaier, G. A. \& Roberts, P. H., 1995a. A three-dimensional convective dynamo solution with rotating and finitely conducting inner core and mantle, Phys. Earth Planet. Inter., 91(1-3), 63-75.

Glatzmaier, G. A. \& Roberts, P. H., 1995b. A three-dimensional selfconsistent computer-simulation of a geomagnetic-field reversal, Nature, 377(6546), 203-209.

Glatzmaier, G. A., Coe, R. S., Hongre, L., \& Roberts, P. H., 1999. The role of the Earth's mantle in controlling the frequency of geomagnetic reversals, Nature, 401(6756), 885-890.

Hawkins, L. M. A., Anwar, T., Shcherbakova, V. V., Biggin, A. J., Kravchinsky, V. A., Shatsillo, A. V., \& Pavlov, V. E., 2019. An exceptionally weak Devonian geomagnetic field recorded by the Viluy Traps, Siberia, Earth Planet. Sci. Lett., 506, 134-145.

Heimpel, M. H. \& Evans, M. E., 2013. Testing the geomagnetic dipole and reversing dynamo models over Earth's cooling history, Phys. Earth Planet. Inter., 224, 124-131.

Heimpel, M. H., Aurnou, J. M., Al-Shamali, F., \& Gomez-Perez, N., 2005. A numerical study of dynamo action as a function of spherical shell geometry, Earth Planet. Sci. Lett., 236(1-2), 542-557.

Hollerbach, R. \& Jones, C. A., 1993. Influence of the Earth's inner core on geomagnetic fluctuations and reversals, Nature, 365(6446), 541-543.

Hulot, G. \& Gallet, Y., 2003. Do superchrons occur without any palaeomagnetic warning?, Earth Planet. Sci. Lett., 210(1-2), 191-201.

Hulot, G. \& Le Mouël, J.-L., 1994. A statistical approach to the Earth's main magnetic field, Phys. Earth Planet. Inter, 82(3-4), 167-183.

Jacobs, J. A., 1953. The Earth's Inner Core, Nature, 172(4372), 297-298.

King, E. M. \& Buffett, B. A., 2013. Flow speeds and length scales in geodynamo models: The role of viscosity, Earth Planet. Sci. Lett., 371372(C), 156-162.

Labrosse, S., 2015. Thermal evolution of the core with a high thermal conductivity, Phys. Earth Planet. Inter., 247, 36-55.

Labrosse, S., Poirier, J.-P., \& Le Mouël, J.-L., 2001. The age of the inner core, Earth Planet. Sci. Lett., 190(3-4), 111-123.

Landeau, M., Aubert, J., \& Olson, P. L., 2017. The signature of inner-core nucleation on the geodynamo, Earth Planet. Sci. Lett., 465, 193-204.

Lhuillier, F. \& Gilder, S. A., 2013. Quantifying paleosecular variation: Insights from numerical dynamo simulations, Earth Planet. Sci. Lett., 382, 87-97.

Lhuillier, F., Aubert, J., \& Hulot, G., 2011a. Earth's dynamo limit of predictability controlled by magnetic dissipation, Geophys. J. Int., 186(2), $492-508$.

Lhuillier, F., Fournier, A., Hulot, G., \& Aubert, J., 2011b. The geomagnetic secular-variation timescale in observations and numerical dynamo models, Geophys. Res. Lett., 38(9).

Lhuillier, F., Hulot, G., \& Gallet, Y., 2013. Statistical properties of reversals and chrons in numerical dynamos and implications for the geodynamo, Phys. Earth Planet. Inter, 220, 19-36.

McFadden, P. L. \& Merrill, R. T., 1984. Lower Mantle Convection and Geomagnetism, J. Geophys. Res, 89(NB5), 3354-3362.

McFadden, P. L. \& Merrill, R. T., 2000. Evolution of the geomagnetic reversal rate since 160 Ma: Is the process continuous?, J. Geophys. Res, 105(B12), 28455-28460.

Nimmo, F., 2015. 8.02 Energetics of the Core, in Core Dynamics, pp. 27-55, eds Olson, P. L. \& Schubert, G., Elsevier B.V.

Olson, P. L., 2007. Gravitational dynamos and the low-frequency geomagnetic secular variation, Proc. Natl. Acad. Sci. U.S.A, 104(51), 2016020166.

Olson, P. L. \& Amit, H., 2014. Magnetic reversal frequency scaling in dynamos with thermochemical convection, Phys. Earth Planet. Inter, 229(C), 122-133

Olson, P. L., Coe, R. S., Driscoll, P. E., Glatzmaier, G. A., \& Roberts, P. H., 2010. Geodynamo reversal frequency and heterogeneous coremantle boundary heat flow, Phys. Earth Planet. Inter., 180(1-2), 66-79.

Olson, P. L., Deguen, R., Hinnov, L. A., \& Zhong, S., 2013. Controls on geomagnetic reversals and core evolution by mantle convection in the Phanerozoic, Phys. Earth Planet. Inter., 214, 87-103.

Olson, P. L., Deguen, R., Rudolph, M. L., \& Zhong, S., 2015. Core evolution driven by mantle global circulation, Phys. Earth Planet. Inter, 243 IS - , 44-55.

Oruba, L. \& Dormy, E., 2014. Transition between viscous dipolar and inertial multipolar dynamos, Geophys. Res. Lett., 41, 7115-7120.

Pavlov, V. E. \& Gallet, Y., 2001. Middle Cambrian high magnetic reversal frequency (Kulumbe River section, northwestern Siberia) and reversal behaviour during the Early Palaeozoic, Earth Planet. Sci. Lett., 185(12), 173-183.

Pavlov, V. E. \& Gallet, Y., 2005. A third superchron during the Early Paleozoic, Episodes, 28(2), 78-84.

Pavlov, V. E. \& Gallet, Y., 2010. Variations in geomagnetic reversal frequency during the Earth's middle age, Geochem. Geophys. Geosyst., 11(1), Q01Z10.

Roberts, P. H. \& Glatzmaier, G. A., 2001. The geodynamo, past, present and future, Geophys. Astrophys. Fluid Dyn., 94(1-2), 47-84.

Shatsillo, A. V., Kuznetsov, N. B., Pavlov, V. E., Fedonkin, M. A., Priyatkina, N. S., Serov, S. G., \& Rudko, S. V., 2015. The first magnetostratigraphic data on the stratotype of the Lopata Formation, Northeastern Yenisei Ridge: Problems of its age and paleogeography of the Siberian Platform at the Proterozoic-Phanerozoic boundary, Dokl. Earth Sc., $\mathbf{4 6 5}(2), 1211-1214$.

Shcherbakova, V. V., Biggin, A. J., Veselovskiy, R. V., Shatsillo, A. V., Hawkins, L., Shcherbakov, V. P., \& Zhidkov, G. V., 2017. Was the Devonian geomagnetic field dipolar or multipolar? Palaeointensity studies of Devonian igneous rocks from the Minusa Basin (Siberia) and the Kola Peninsula dykes, Russia, Geophys. J. Int., 209(2), 1265-1286.

Smirnov, A. V., Tarduno, J. A., Kulakov, E. V., McEnroe, S. A., \& Bono, R. K., 2016. Palaeointensity, core thermal conductivity and the unknown age of the inner core, Geophys. J. Int., 205(2), 1190-1195.

Stelzer, Z. \& Jackson, A., 2013. Extracting scaling laws from numerical dynamo models, Geophys. J. Int., 193(3), 1265-1276.

Tauxe, L. \& Yamazaki, T., 2007. Paleointensities, in Geomagnetism, pp. 509-563, eds Kono, M. \& Schubert, G., Elsevier, Amsterdam.

Tivey, M. A., Sager, W. W., Lee, S.-M., \& Tominaga, M., 2006. Origin of the Pacific Jurassic quiet zone, Geology, 34(9), 789.

Valet, J.-P. \& Fournier, A., 2016. Deciphering records of geomagnetic reversals, Rev. Geophys., 54.

Wicht, J., 2002. Inner-core conductivity in numerical dynamo simulations, Phys. Earth Planet. Inter., 132(4), 281-302.

Wicht, J., 2005. Palaeomagnetic interpretation of dynamo simulations, Geophys. J. Int., 162(2), 371-380.

Wicht, J. \& Meduri, D. G., 2016. A gaussian model for simulated geomagnetic field reversals, Phys. Earth Planet. Inter, 259, 45-60. 